\title{
Interpretations of gamma-ray burst spectroscopy
}

\section{Bright BATSE bursts}

\author{
F. Ryde ${ }^{1}$, D. Kocevski ${ }^{2}$, Z. Bagoly ${ }^{3}$, N. Ryde ${ }^{4}$, and A. Mészáros ${ }^{1,5}$ \\ 1 Stockholm Observatory, AlbaNova University Center, 10691 Stockholm, Sweden \\ e-mail: felix@astro.su.se \\ 2 Department of Physics and Astronomy, Rice University, Houston, TX 77005, USA \\ 3 Laboratory for Information Technology, Eötvös University, Budapest 1117, Hungary \\ ${ }^{4}$ Uppsala Astronomical Observatory, Box 515, 75120 Uppsala, Sweden \\ 5 Astronomical Institute of the Charles University, 18000 Prague 8, V Holešovičkách 2, Czech Republic
}

Received 30 June 2004 / Accepted 8 November 2004

\begin{abstract}
We analyze the spectral lags of a sample of bright gamma-ray burst pulses observed by CGRO BATSE and compare these with the results of high-resolution spectroscopical investigations. We find that pulses with hard spectra have the largest lags, and that there is a similar, but weaker correlation between hardness-intensity correlation index, $\eta$, and lag. We also find that the lags differ considerably between pulses within a burst. Furthermore, the peak energy mainly decreases with increasing lag. Assuming a lag-luminosity relation as suggested by Norris et al., there will thus be a positive luminosity-peak-energy correlation. We also find that the hardness ratio, of the total flux in two channels, only weakly correlates with the spectral evolution parameters. These results are consistent with those found in the analytical and numerical analysis in Paper I. Finally, we find that for these bursts, dominated by a single pulse, there is a correlation between the observed energy-flux, $F$, and the inverse of the lag, $\Delta t: F \propto \Delta t^{-1}$. We interpret this flux-lag relation found as a consequence of the lag-luminosity relation and that these bursts have to be relatively narrowly distributed in $z$. However, they still have to, mainly, lie beyond $z \sim 0.01$, since they do not coincide with the local super-cluster of galaxies. We discuss the observed correlations within the collapsar model, in which the collimation of the outflow varies. Both the thermal photospheric emission as well as non-thermal, optically-thin synchrotron emission should be important.
\end{abstract}

Key words. gamma-rays: bursts

\section{Introduction}

In a previous paper (Ryde 2005; Paper I) we demonstrated the relation between the two approaches used to characterize the spectral evolution in gamma-ray bursts (GRBs): the one using spectral lags between energy channels and the one using high-resolution data, leading to detailed parametrization of the evolution. Specifically, we investigated this relation for individual pulses, using an analytical model and numerical simulations. We showed that the spectral evolution described by high spectral-resolution data, in empirical correlations, naturally leads to correlations involving spectral lags. The relation between the analyses is not trivial and a general description can only be given approximately, but can be given exactly case by case. This is because the lag measures a combination of spectral evolution parameters. It further depends on the relative channel widths the data are divided into.

We will here briefly summarize the main results of the analytical and numerical simulation analysis from Paper I. We found that the spectral lag correlates strongest with the decay time-scale of the pulse (or equivalently of the peak-energy decay). Bursts with hard spectra, that is, with large low-energy spectral power-law slope, $\alpha$, exhibit the largest lags. Similarly, a more energetic burst, as measured by the peak energy of the spectra, also, most often, yields a large lag. However, for very soft bursts the relation is the opposite. Furthermore, the ratio of the flux in two different energy channels, the hardness ratio, is found to only weakly vary with $\alpha$ and $\eta$ (coefficient of the hardness-intensity correlation, HIC, see Eq. (1) in Paper I), and it is mainly determined by how energetic the burst is. Characteristic differences in the behavior of hard and soft pulses are identified, as well as differences in behavior between more and less energetic bursts. Further, we could also reproduce the observation by Kocevski \& Liang (2003), namely a good correlation between lag and the quantity $\Phi_{0}$ normalized by the peak flux, $=\Phi_{0} / F$. The parameter $\Phi_{0}$ describes the decay rate of the peak energy of the spectrum (see Eq. (2), in Paper I). For different pulses within a burst, the spectral parameters are, in general, not the same, and thus the lag is not expected to be the same during the whole bursts. 
In this paper we will analyze GRB pulses, observed by the Burst and Transient Source Experiment (BATSE) on the Compton Gamma-Ray Observatory (CGRO), and compare the results with the trends and correlations summarized above. This will be done in Sect. 2. In particular, we will study the spectral lags and hardness ratios. In Sect. 3, we will also discuss how our results relate to the physical models that have been proposed to explain the observed lag correlations. We conclude our discussion in Sect. 4

\section{CGRO BATSE observations}

We defined a sample of strong and well-separated pulses by using the Borgonovo \& Ryde (2001) sample which consists of 47 pulses that all show a clear spectral evolution defined by the hardness-intensity correlation (HIC) during the pulse decay. From these we chose the cases which have a "clean" rise phase that is not contaminated greatly by previous pulses. We also limited our sample to cases for which the rise time, $t_{\mathrm{r}}$, is much shorter than the decay time, $t_{\mathrm{d}}: t_{\mathrm{r}} / t_{\mathrm{d}}<0.2$. This gave us the sample of 17 pulses in 15 bursts, which are presented in Table 1 . We analyzed the four-channel discriminator rates of BATSE and as customary, used channel $1(\sim 25-50 \mathrm{keV})$ and channel $3(\sim 100-300 \mathrm{keV})$. These data are constructed from three data types from the Large Area Detector (LAD): DISCLA, PREB, and DISCSC (Fishman 1989). We also analyzed the 128-channel data (high energy-resolution data, HERB), to be able to compare the results. We deconvolve the observed count data into photon fluxes and performed the analysis with RMFIT 1.0b6, provided by the BATSE team at MSFC (see e.g. Ryde \& Svensson 2000 for details).

\subsection{Analysis of pulses}

In Paper I, we derived an analytical function for a GRB pulse, with four parameters (see also Kocevski et al. 2003):

$F(t)=\frac{A_{0}\left(1-\mathrm{e}^{-t / t_{\mathrm{r}}}\right)}{\left(1+\left(t+t_{\mathrm{r}} \mathrm{e}^{-t / t_{\mathrm{r}}}\right) / \tau\right)^{d}}$.

Here $\tau$ is the decay time scale and $t_{\mathrm{r}}$ is connected to the rise phase. The decay index $d$ is defined by requiring that $F(t) \rightarrow$ $t^{-d}$ as $t \rightarrow \infty$ and $A_{0}$ is an analytical function of the other parameters. We will also use the peak energy, $E_{0}$, at the beginning of the pulse, which is given when $t=0$ in Eq. (1) and defines the onset of pulse emission according to the analytical model. The channel light-curves in our sample were fitted with this function. Figure 1 depicts a few examples of the studied bursts and their channels 1 and 3 light curves and the fits are shown by solid lines. Even though the function was deduced for the bolometric light curve, it is flexible enough to fit the channel light-curves, which is reflected by the fact that the fits mainly have good values of the $\chi^{2}$. In Col. 2 of Table 2 the peak times are given for the light curves over the whole spectral range of BATSE ( 25-2000 keV). The peak time is given in seconds since the BATSE trigger, and is thus different from $t$ in Eq. (1) by a constant offset. The main purpose of measuring this time is to compare it with the same time measured on the photon light-curves instead (see Sect. 2.3)
Table 1. Sample of 17 pulses in 15 GRBs defined by their peak times.

\begin{tabular}{lccc}
\hline \hline Burst & Trigger & LAD & $t_{\max }[\mathrm{s}]$ \\
\hline GRB 911016 & 907 & 1 & 1.6 \\
GRB 911031 & 973 & 3 & $2.8 / 24$ \\
GRB 911104 & 999 & 2 & 4.0 \\
GRB 920525 & 1625 & 4 & 4.9 \\
GRB 920830 & 1883 & 0 & 1.2 \\
GRB 921207 & 2083 & 0 & 8.6 \\
GRB 930201 & 2156 & 1 & 14 \\
GRB 941026 & 3257 & 0 & 3.0 \\
GRB 950624 & 3648 & 3 & $23 / 41$ \\
GRB 950818 & 3765 & 1 & 66 \\
GRB 951102 & 3891 & 2 & 33 \\
GRB 960530 & 5478 & 2 & 2.0 \\
GRB 960804 & 5563 & 4 & 1.4 \\
GRB 980125 & 6581 & 0 & 48 \\
GRB 990102 & 7293 & 6 & 3.2 \\
\hline
\end{tabular}

\subsubsection{Analytic lags, lag13}

For every pulse, the analytic spectral lag, $\equiv \operatorname{lag} 13$, was found by measuring the time difference between the light curve peaks in channels 1 and 3 . These peaks were identified as the maxima of the function in Eq. (1). These results are given in the third column in Table 2 . We note that there is a large spread in values of the lag. The largest value is for the first pulse in GRB 960524 (BATSE trigger \# 3648) with 2.65 s. Furthermore, GRB 980125 (\# 6581) and the second pulse in GRB 911031 (\# 973) are insignificantly different from zero, which means that the pulses in the four spectral channels peak at the same time. As noted by Band (1997) and Norris et al. (2000), GRB lags are, in general, concentrated towards such short time scales $(<100 \mathrm{~ms})$. The case GRB 951102 (\# 3891) is an example of a pulse with a negative lag, that is, the hardest radiation lags behind the softer radiation, even though the significance of it being different from zero is low. Finally, the lags for the two cases in the sample which have two pulses GRB 911031 (\# 973) and GRB 960524 (\# 3648) show that the sizes of the lags are significantly different between the pulses. This is an important observation, since most often lags are measured over the whole burst and it is assumed that the lag is constant. Such a lag measurement therefore more reflects the averaged lag behavior. Furthermore, Borgonovo \& Ryde (2001) noted that the HIC index, $\eta$, in multi-pulse bursts are often constant from pulse to pulse, while Liang \& Kargatis (1996) noted that the HFC index, $\Phi_{0}$, is similarly, practically constant. Both these observations are in contrast to that of the lags, which do differ significantly. F. Ryde \& D. Kocevski (2005, in prep.) have made a more detailed analysis of the spectral lag in multi-pulsed bursts and showed that this is a general feature. 

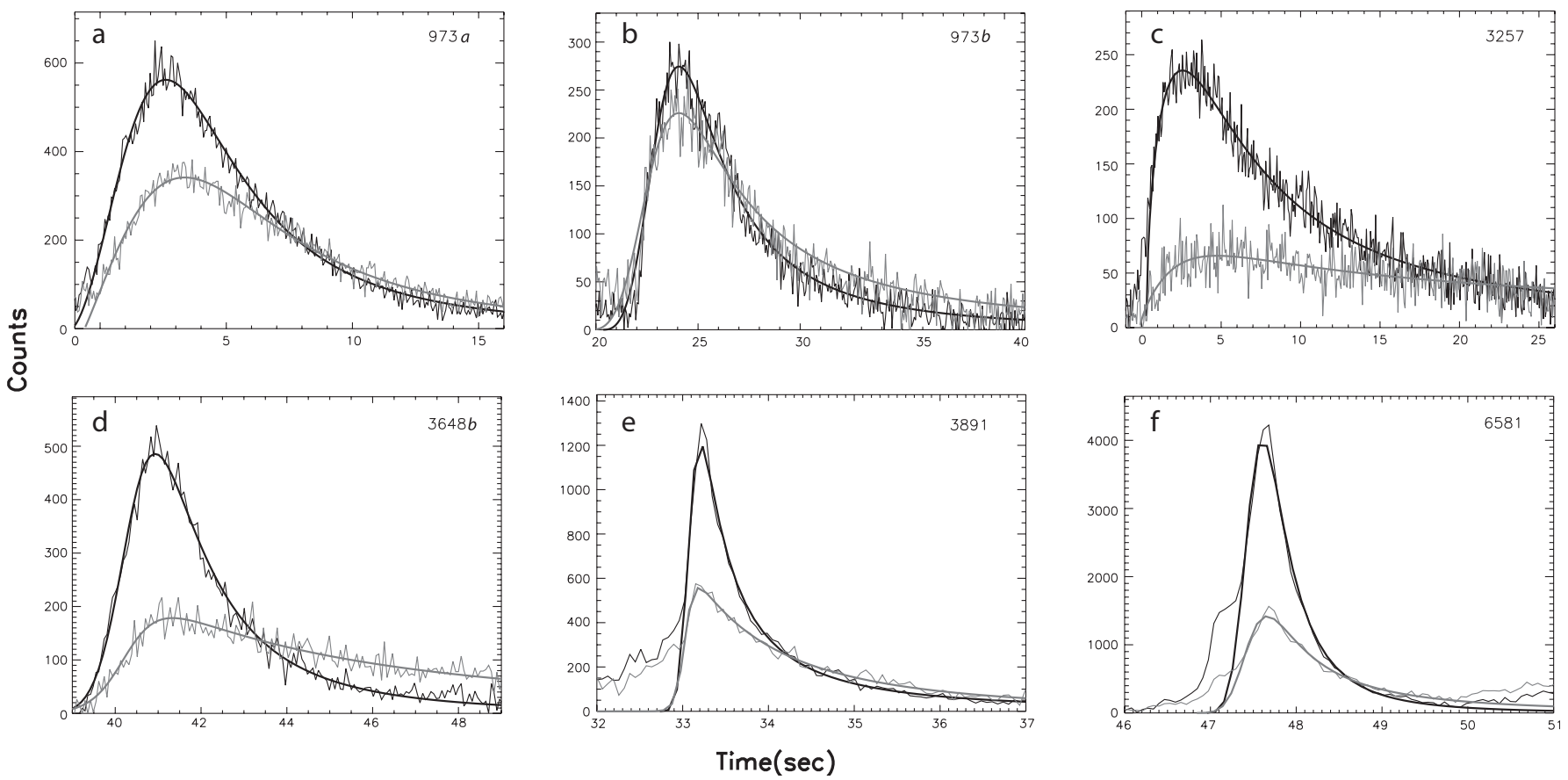

Fig. 1. Light-curves of six of the studied pulses; a) GRB 911031 (\# 973a); b) GRB 911031 (\# 973b); c) GRB 941026 (\# 3257 ); d) GRB 960524 (\# 3648); e) GRB 951102 (\# 3891); f) GRB 980125 (\# 6581). The black curves are for BATSE channel 3 and the grey curves for channel 1. The smooth line is the best fit model (Eq. (1)).

Table 2. The peak times and lags of the pulses in the sample.

\begin{tabular}{lccccc}
\hline \hline & \multicolumn{2}{c}{ Counts } & & \multicolumn{2}{c}{ Photon flux } \\
\cline { 2 - 3 } \cline { 5 - 6 } Trigger & Peak time $[\mathrm{s}]$ & lag $13[\mathrm{~s}]$ & & Peak time $[\mathrm{s}]$ & lag13 [s] \\
\hline 907 & $1.55 \pm 0.01$ & $1.3 \pm 0.3$ & & $2.1 \pm 0.1$ & $3.1 \pm 1.6$ \\
$973 \mathrm{a}$ & $2.84 \pm 0.02$ & $0.75 \pm 0.08$ & & $3.1 \pm 0.1$ & $0.8 \pm 0.6$ \\
$973 \mathrm{~b}$ & $24.04 \pm 0.03$ & $.0004 \pm 0.06$ & & $24.1 \pm 0.2$ & $0.1 \pm 0.7$ \\
999 & $3.939 \pm 0.003$ & $0.11 \pm 0.01$ & & $4.02 \pm 0.02$ & $0.14 \pm 0.11$ \\
1625 & $4.859 \pm 0.005$ & $0.15 \pm 0.02$ & & $4.98 \pm 0.03$ & $0.12 \pm 0.16$ \\
1883 & $1.23 \pm 0.02$ & $0.9 \pm 0.1$ & & $1.59 \pm 0.09$ & $1.2 \pm 0.4$ \\
2083 & $8.578 \pm 0.004$ & $0.23 \pm 0.03$ & & $8.67 \pm 0.03$ & $0.2 \pm 0.2$ \\
2156 & $14.45 \pm 0.01$ & $0.27 \pm 0.05$ & & $14.64 \pm 0.05$ & $0.3 \pm 0.3$ \\
3257 & $2.944 \pm 0.001$ & $2.2 \pm 0.8$ & & $4.5 \pm 0.3$ & $3 \pm 5$ \\
$3648 \mathrm{a}$ & $23.5 \pm 0.1$ & $2.7 \pm 0.6$ & & $23.9 \pm 0.4$ & $1.7 \pm 4.3$ \\
$3648 \mathrm{~b}$ & $40.99 \pm 0.01$ & $0.4 \pm 0.1$ & & $41.2 \pm 0.1$ & $0.7 \pm 0.8$ \\
3765 & $66.069 \pm 0.004$ & $0.05 \pm 0.02$ & & $66.12 \pm 0.02$ & $0.05 \pm 0.11$ \\
3891 & $33.198 \pm 0.005$ & $-0.02 \pm 0.03$ & & $33.24 \pm 0.02$ & $-0.03 \pm 0.11$ \\
5478 & $2.00 \pm 0.05$ & $0.8 \pm 0.3$ & & $2.3 \pm 0.2$ & $1.3 \pm 1.7$ \\
5563 & $1.418 \pm 0.004$ & $0.07 \pm 0.01$ & & $1.47 \pm 0.01$ & $0.08 \pm 0.06$ \\
6581 & $47.607 \pm 0.002$ & $0.053 \pm 0.009$ & & $47.66 \pm 0.02$ & $0.08 \pm 0.04$ \\
7293 & $3.24 \pm 0.06$ & $2.0 \pm 0.9$ & & $4.0 \pm 0.3$ & $3 \pm 4$ \\
\hline
\end{tabular}

\subsubsection{Fluxes and hardness ratios}

We also calculated the photon flux $\left[\mathrm{s}^{-1} \mathrm{~cm}^{-2}\right]$ at the peak of the light curve, $F_{\mathrm{pk}}$, which appears in Col. 6 in Table 3. In Fig. 2, this flux is plotted versus the "analytic spectral lag", lag13. There is one data point that differs considerably from the power-law correlation that appears, and that is for the second pulse in trigger 973. Excluding this point, the best fit to the correlation is

$F_{\mathrm{pk}}=5.44 \Delta t^{-0.91 \pm 0.05}$.

This relation is reminiscent of the lag-luminosity relation found by Norris et al. (2000) and the corresponding relation for the photon flux Salmonson (2000) and will be discussed further in Sect. 3.1. The fact that the second pulse in 973 does not follow 
Table 3. The HRS parameters for the studied sample.

\begin{tabular}{|c|c|c|c|c|c|c|c|}
\hline $\begin{array}{l}\text { BATSE } \\
\text { Trigger }\end{array}$ & $\eta$ & $\langle\alpha\rangle$ & $\begin{array}{c}E_{0} \\
{[\mathrm{keV}]}\end{array}$ & $\begin{array}{c}\Phi_{0} \\
{\left[\mathrm{~cm}^{-2}\right]}\end{array}$ & 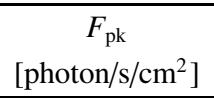 & $\overline{H R}$ & $z^{1}$ \\
\hline 907 & $2.34 \pm 0.15$ & $0.37 \pm 0.08$ & $504 \pm 75$ & $20 \pm 1$ & $4.9 \pm 0.3$ & 1.87 & 0.40 \\
\hline $973 a$ & $1.45 \pm 0.21$ & $-1.01 \pm 0.03$ & $515 \pm 25$ & $55 \pm 11$ & $12.4 \pm 0.7$ & 1.30 & 0.35 \\
\hline $973 b$ & $0.84 \pm 0.1$ & $-1.38 \pm 0.06$ & $544 \pm 58$ & $17 \pm 4$ & $6.8 \pm 0.2$ & 0.97 & $1.7^{3}$ \\
\hline 999 & $1.9 \pm 0.1$ & $-0.5 \pm 0.1$ & $373 \pm 35$ & $24 \pm 1$ & $20.9 \pm 1.5$ & 1.81 & 0.67 \\
\hline 1625 & $2.37 \pm 0.3$ & $-0.74 \pm 0.02$ & $1005 \pm 45$ & $42 \pm 7$ & $55.3 \pm 1.1$ & 2.35 & 1.8 \\
\hline $1883^{2}$ & $2.39 \pm 0.29$ & $-0.3--2.0$ & $451 \pm 21$ & $18 \pm 1$ & $8.7 \pm 0.8$ & 1.23 & 0.45 \\
\hline 2083 & $1.78 \pm 0.04$ & $-0.36 \pm 0.02$ & $1010 \pm 60$ & $65 \pm 2$ & $86.0 \pm 1.5$ & 0.55 & 0.18 \\
\hline 2156 & $1.55 \pm 0.15$ & $-0.84 \pm 0.02$ & $475 \pm 65$ & $42 \pm 6$ & $23.1 \pm 0.7$ & 2.08 & 0.41 \\
\hline 3257 & $2.8 \pm 0.2$ & $0.07 \pm 0.06$ & $344 \pm 22$ & $75 \pm 4$ & $3.8 \pm 0.4$ & 1.65 & 0.38 \\
\hline $3648 \mathrm{a}$ & $0.64 \pm 0.03$ & $-0.61 \pm 0.2$ & $157 \pm 19$ & $19 \pm 1$ & $1.5 \pm 0.4$ & 0.53 & $1.11^{3}$ \\
\hline $3648 b$ & $1.42 \pm 0.08$ & $-0.36 \pm 0.05$ & $716 \pm 17$ & $19 \pm 2$ & $8.5 \pm 0.4$ & 1.28 & 0.38 \\
\hline 3765 & $2.4 \pm 0.2$ & $-0.76 \pm 0.02$ & $381 \pm 17$ & $64 \pm 8$ & $45 \pm 2$ & 1.86 & 0.64 \\
\hline 3891 & $1.8 \pm 0.18$ & $-0.75 \pm 0.06$ & $269 \pm 13$ & $17 \pm 1$ & $29.6 \pm 1.2$ & 1.36 & 0.68 \\
\hline 5478 & $1.87 \pm 0.16$ & $0.13 \pm 0.13$ & $813 \pm 32$ & $17 \pm 2$ & $3.8 \pm 0.9$ & 1.30 & 0.53 \\
\hline 5563 & $2.35 \pm 0.3$ & $-0.87 \pm 0.07$ & $320 \pm 15$ & $19 \pm 4$ & $39 \pm 2$ & 1.35 & 0.76 \\
\hline 6581 & $1.62 \pm 0.2$ & $-0.65 \pm 0.03$ & $762 \pm 40$ & $20 \pm 1$ & $87.2 \pm 1.5$ & 2.04 & 1.16 \\
\hline 7293 & $2.04 \pm 0.12$ & $0.79 \pm 0.09$ & $397 \pm 51$ & $38 \pm 4$ & $3.7 \pm 0.5$ & 1.97 & 8.6 \\
\hline
\end{tabular}

\footnotetext{
${ }^{1}$ Lag-estimated.
}

${ }^{2}$ Strong $\alpha$-evolution.

${ }^{3}$ Secondary pulse; ignored.

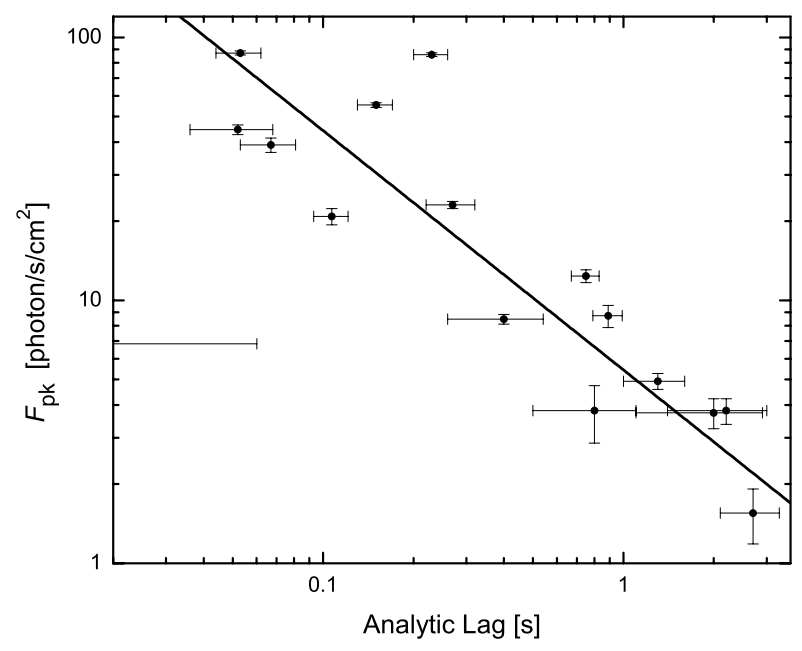

Fig. 2. Peak photon flux as a function of spectral lag. The best fit is marked by the solid line and approximately corresponds to an inverse relation between flux and lag.

the general trend is of interest. As mentioned above the lags for different pulses vary within a burst. Therefore, the relation in Eq. (2) (or correspondingly, the lag-luminosity relation) could either be valid for all individual pulses, each of which produces a point following the correlation, or be valid only for the dominant pulse in a burst. The latter alternative was promoted by Hakkila \& Giblin (2004) who discussed two bursts, of which a later emission component had a significantly different lag. Also by removing the low-intensity emission in calculating the CCF lags, Norris et al. (2000) disregarded any differences in lag for the weaker pulses. The behavior of the second pulse in trigger 973, studied here, suggests such an interpretation as well, that is, it is mainly the dominant pulse that gives rise to the correlation.

Finally, we calculated the hardness ratio (HR31), defined as the ratio of the total counts in BATSE channels 3 and 1 . We integrated the count light-curves in the interval which had a flux level above $10 \%$ of the peak value. The ratios are given in Col. 7 in Table 3.

All these measurement will be compared with the highresolution spectroscopical analysis below (Sect. 2.4). However, before doing this, we will investigate two issues that could affect the interpretation, namely the use of the cross correlation function as a measure of the spectral lag (Sect. 2.2) and the use of count light curves (Sect. 2.3).

\subsection{The cross correlation function, CCF13}

It is often the case, for low signal-to-noise bursts, that a proper deconvolution cannot be made. Problems in fitting the channel light-curves with analytical functions also arise for weak bursts. Therefore, the cross correlation function (CCF), measured on the count light-curves, has to be used to characterize the shifts in time. For BATSE observations the spectral lag $^{1}$ is often calculated between channels $1(\sim 25-50 \mathrm{keV})$ and $3(\sim 100-300 \mathrm{keV})$. As the light curves (or time series) are

1 Formally the lag is the variable of the correlation function and therefore a better notation of the time-shift between the pulses is the peak lag. 


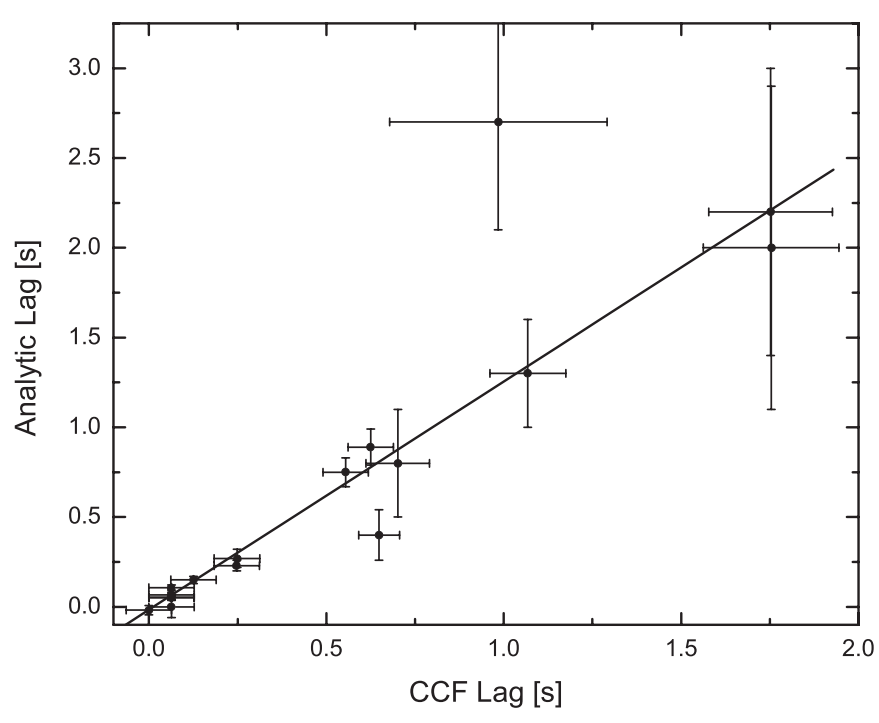

Fig. 3. Analytic spectral lag, $\Delta t$, versus the CCF lag CCF13. A good linear correspondence exists, as expected.

sampled at a number of discrete data points sums are calculated instead of integrals (see Eq. (4) in Paper I). The discrete CCF analysis was implemented by methods similar to those employed by Band (1997) and Norris et al. (2000) and we denote this measured lag by $C C F 13$. We therefore need to investigate whether there is any difference in the measurements between analytic lags, that is $\operatorname{lag} 13$, and $C C F 13$.

We calculated the $\operatorname{CCF}(\Delta t, \operatorname{ch} 1, \operatorname{ch} 3)$ and found its maximum value, to identify the spectral lag, $\Delta t$, between the light curves in channels 1 and 3 . We fitted a cubic polynomial to the peak of the resulting discrete CCF function. The statistical error in this measurement was estimated by using a Monte Carlo routine in which Poisson distributed noise was added to both channels individually by an amount consistent with the observed signal-to-noise ratio. Each iteration produced an independent lag measurement, allowing for the accumulation of a cross-correlation peak-distribution (CCPD). This process was continued until the resulting CCPD approached a normal distribution, which typically took $250-500$ runs. To this resulting distribution a Gaussian function was fit from which the peak determines the most likely lag and the full-width half-max yields the $1 \sigma$ confidence level. The results are also given in Table 4. In Fig. 3 the analytic lag, lag13, is plotted as a function of the CCF lag, CCF13. A linear fit is given which shows that there is a good correspondence between the two methods. The best fit is $\operatorname{lag} 13=(-0.02 \pm 0.01)+(1.27 \pm 0.08) \times C C F 13$. The $\mathrm{CCF}$ lag measurements thus tend to underestimate the real time difference between the peaks of the light curves in the two energy bands. The bursts in our sample are simple pulse structures and therefore the correspondence might be better than would be the case for very variable light curves. Wu \& Fenimore (2000) pointed out that it is not always reliable to determine lags with CCF. This is especially the case for multi-peaked bursts, for which both the HR and the CCF give average, quantitative descriptions. They also noted that the methods used to calculate the CCFs can affect the results considerably. For instance, the inclusion of time intervals when the signal is at background
Table 4. Spectral lags between channels 1 and 3 measured by their light curves (lag13) and with the CCF (CCF31).

\begin{tabular}{lcc}
\hline \hline & \multicolumn{2}{c}{ Spectral lags } \\
\cline { 2 - 3 } Trigger & $1.3 \pm 13[\mathrm{~s}]$ & CCF31 [s] \\
\hline 907 & $0.75 \pm 0.08$ & $0.07 \pm 0.11$ \\
$973 \mathrm{a}$ & $4 \times 10^{-4} \pm 0.06$ & $0.06 \pm 0.06$ \\
$973 \mathrm{~b}$ & $0.11 \pm 0.01$ & $0.06 \pm 0.06$ \\
999 & $0.15 \pm 0.02$ & $0.13 \pm 0.06$ \\
1625 & $0.9 \pm 0.1$ & $0.63 \pm 0.06$ \\
1883 & $0.23 \pm 0.03$ & $0.25 \pm 0.06$ \\
2083 & $0.27 \pm 0.05$ & $0.25 \pm 0.06$ \\
2156 & $2.2 \pm 0.8$ & $1.8 \pm 0.2$ \\
3257 & $2.7 \pm 0.6$ & $1.0 \pm 0.3$ \\
$3648 \mathrm{a}$ & $0.4 \pm 0.1$ & $0.65 \pm 0.06$ \\
$3648 \mathrm{~b}$ & $0.05 \pm 0.02$ & $0.06 \pm 0.06$ \\
3765 & $-0.02 \pm 0.03$ & $0.00 \pm 0.06$ \\
3891 & $0.8 \pm 0.3$ & $0.70 \pm 0.09$ \\
5478 & $0.07 \pm 0.01$ & $0.06 \pm 0.06$ \\
5563 & $0.053 \pm 0.009$ & $0.06 \pm 0.06$ \\
6581 & $2.0 \pm 0.9$ & $1.8 \pm 0.2$ \\
7293 & &
\end{tabular}

will clearly affect the measurements. What we find here is that the CCF measurement represents well the actual lag for the smooth pulses in our sample. Finally, the CCF13 for the first and second pulses in 973 and the second and third pulses in 3648 are again notably different, reconfirming the conclusion above.

\subsection{Detector counts}

We now turn to the fact that the spectral lag analysis is made straight on the count rate data, which is the method commonly used in the literature. This introduces an important caveat since, in general, the count light-curves have a time-dependent relation to the photon (or energy) flux light-curves. The probability of detecting an incoming photon is energy dependent and it therefore becomes time dependent if the energy distribution of photons vary with time, that is, if there is a significant spectral evolution. For instance, for the BATSE Large Area Detector (LAD) the probability of obtaining a count for an incoming photon is near zero at and below $20 \mathrm{keV}$ and at $30 \mathrm{keV}$ it rises steeply. From 50 to $300 \mathrm{keV}$ (and higher) the probability of a count approaches unity. In the same manner, the energy measurement attached to the count will be less correct, the higher energy the incoming photon has; the photon is less likely to leave $100 \%$ of its energy in the detector. So, to be able to interpret the count data in a meaningful way, one therefore has to assume the spectral evolution has negligible effect. Indeed, assuming no spectral evolution during the pulse, there would be a constant correspondence between the count rate in BATSE channels $2+3$ and photon flux $50-300 \mathrm{keV}$ for each GRB. For a burst with a different spectrum, the constant would be different. However, significant spectral evolution does take 
place during pulses (see, e.g. Ryde 1999) and therefore the count-rate light-curve could result in a misleading interpretation. Note that all these effects are well-known and are included in the detector response matrices and are considered when the deconvolutions are made.

We will therefore redo the analytic-lag analysis on the photon-flux light-curves instead, but still in the same energy bands, to see how the peak times and the lags are affected. To be able to do this we need to deconvolve the detected count fluxes through the detector response. The pulses in our sample are strong enough to allow us to do this without reducing the high time-resolution of the $\sim 64 \mathrm{~ms}$ DISCSC data. The detected spectra were deconvolved by using the empirical GRB model (Band et al. 1993), which consists of two power laws, smoothly joined together. The high-energy power-law, $\beta$, was fixed to the averaged value, while the low-energy power-law, $\alpha$, was left free to vary, whenever permitted by the quality of the data. The purpose of the fits is to make the deconvolution and not to find the exact parameter values and therefore we can allow quite low signal-to-noise ratios. Finally, to arrive at the photon-flux light curve, we integrate the photon spectra over the relevant energy bands.

The comparison is shown in Table 2. Columns 4 and 5 contain the same information as the previous two, but now they are measured on the photon light-curves instead. In Col. 4 are given the peak times for the light-curves, integrated over the the four energy channels, and in Col. 5 are given the analytic lags. The first conclusion to be drawn from the table is that the peak times are systematically underestimated by using the count light-curves compared to the deconvolved light-curves. The relative difference, $\left(t_{\mathrm{c}}-t_{\mathrm{p}}\right) / t_{\mathrm{c}}$, is, in general, some 10 per cent, and in some cases much higher. Also the photon lags are, in general, somewhat larger and they also exhibit a slightly broader dispersion. However, even though there are noticeable differences they are not alarming, and especially considering that larger errors are introduced by the deconvolution process, the conclusion is that the count light-curves, at least for the long pulses ( $>$ few seconds), do give a reasonable value for these studies.

\subsection{Comparison with high-resolution spectroscopy}

We performed the high-spectral resolution spectroscopy on the HERB data following the method outlined in Ryde \& Svensson (2000). We fitted the instantaneous spectra with the highest time resolution permitted by the data. From these fits we calculated the time-averaged, low-energy, power-law index $\langle\alpha\rangle$. The instantaneous $\alpha$ parameter is approximately constant in all except four cases; triggers 999, 1625, 1883, and 2083. For instance, in trigger 1883, $\alpha$ is constant at around -0.3 for the first few seconds and thereafter decreases rapidly to approximately -2 . At the same time $E_{\mathrm{p}}$ decreases to $\sim 30 \mathrm{keV}$. It is therefore likely that the observed variation in $\alpha$ is due to the limited energy band being used and that $E_{\mathrm{p}}$ moves beyond the low energy threshold. The low-energy slope could therefore very well actually be constant for this burst. From the evolution of the instantaneous fits we analyzed the peak-energy evolution, $E_{\mathrm{p}}=E_{\mathrm{p}}(t)$, and the spectral correlations, the hardness-intensity correlation (HIC), $F \propto E_{\mathrm{p}}^{\eta}$ and the hardness-fluence correlation (HFC), $\mathrm{d} E_{\mathrm{p}} / \mathrm{d} t=F / \Phi_{0}$. The resulting parameters for the individual triggers are given in Table 3 and their dependence are shown in Fig. 4. Panel (a) suggests that there is a trend that harder spectra, that is larger $\alpha$, give rise to larger lags. This is in agreement with the numerical simulations in Paper I (their Fig. 3), in which such a trend is indeed found for all $\eta$ values. Panel (b) shows that there is a positive correlation, even though weak, between $\eta$ and $\alpha$; the hard-spectral pulses have the steepest HIC power-laws. These two relations combined suggest that $\eta$ should be positively correlated with lag. Panel (c) shows a weak correlation in this direction. To be able to compare this result with the numerical simulation in Fig. 4 in Paper I, one must recognize the correlation between $\eta$ and $\alpha$. This means that as $\eta$ increases so does $\alpha$ and the relevant panel changes from (d) down to (a). The dominant effect turns out to be the increase in lag due to $\alpha$, which ensures the positive correlation between $\eta$ and lag discussed here. Finally, panel (d) shows a trend that, at least for the large lag cases, lower $E_{0}$ gives the largest lags. The numerical simulation in Paper I (their Fig. 4d) shows that this is the case for very soft-spectra pulses (for all $\eta$ ). Since, for the observed bursts above, $\alpha$ and $\eta$ are correlated (panel (b)), bursts with hard spectra (larger $\alpha$ ) will predominantly have large $\eta$-values. This means that the runs made in Paper I for large $\alpha$ (the earlier panels in Fig. 4 in Paper I; c, b, a in increasing order) are relevant mainly for the large $\eta$-values, i.e., the upper section of those panels. This is indeed where the decrease in lag as a function of $E_{0}$ occurs. This will cause the behavior found in panel (d) of the figure above.

In Paper I we also noted that the hardness ratios only weakly depend on the spectral evolution parameters, since their relations are complicated and the spectral parameters themselves have broad dispersions which will weaken any correlations. This should be most pronounced for low $E_{0}$-values (see Fig. 5 in Paper I). In Fig. 5, we plot the measured HRs and their relationship with the corresponding parameters $E_{0}, \alpha$ and $\eta$. The correlations are indeed weak with only subtle trends. The panel on the left suggests a positive correlation between $E_{0}$ and the HR, which is consistent with the numerical simulations in Paper I (their Figs. 5a and 7). A higher initial peak energy increases the counts in the higher channels and thereby the HRs. The two following panels similarly suggest a positive correlation between HR and the power-law slope, $\alpha$ and the HIC $\eta$, respectively. According to the numerical simulations, harder spectra (steeper HICs) are expected to have larger HR (Figs. 5b(a) in Paper I), even though the trends are, again, expected to be weak. A harder spectrum decreases the relative count flux in the lower channels, thereby increasing the HRs. The HR31- $\alpha$ correlation, combined with the $\alpha-\eta$ correlation in Fig. 4 d, is consistent with the HR31- $\eta$ correlation in the panel on the right.

Finally, in Fig. 6 we plot the lag dependence on $\Phi_{0} / F_{\text {tot }}$. The correlations discussed in Kocevski \& Liang (2003) emerge as expected from the analytical and numerical simulations in Paper I. The power-law index is $0.92 \pm 0.06$, consistent with unity. 

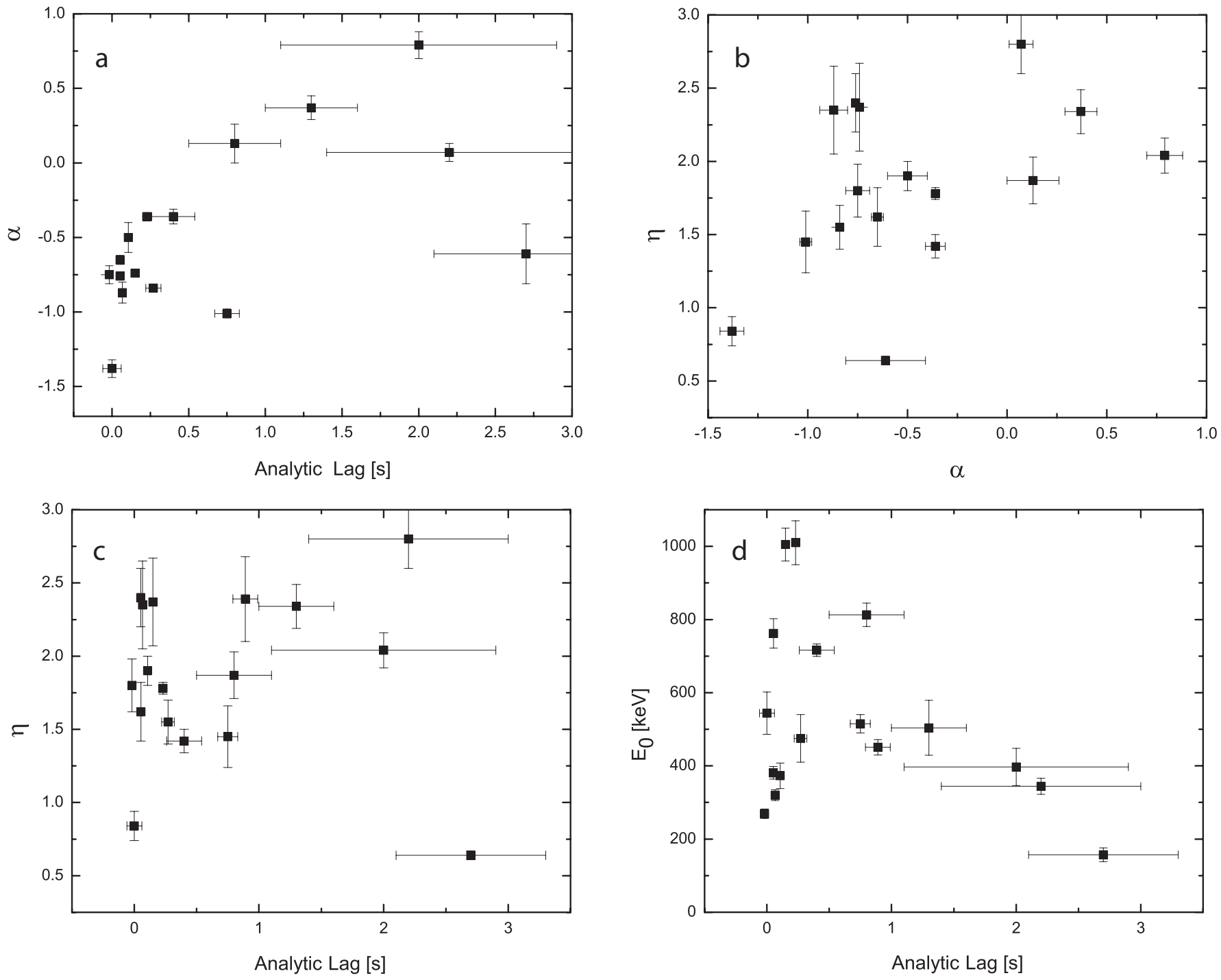

Fig. 4. $C G R O$-BATSE data analyzed using high resolution spectroscopy, HRS. a) Low-energy power-law index, $\alpha$, versus spectral lag. b) HIC power-law index $\eta$, versus $\alpha$. c) $\alpha$ versus lag. d) Initial (maximal) peak-energy, $E_{0}$, versus lag.

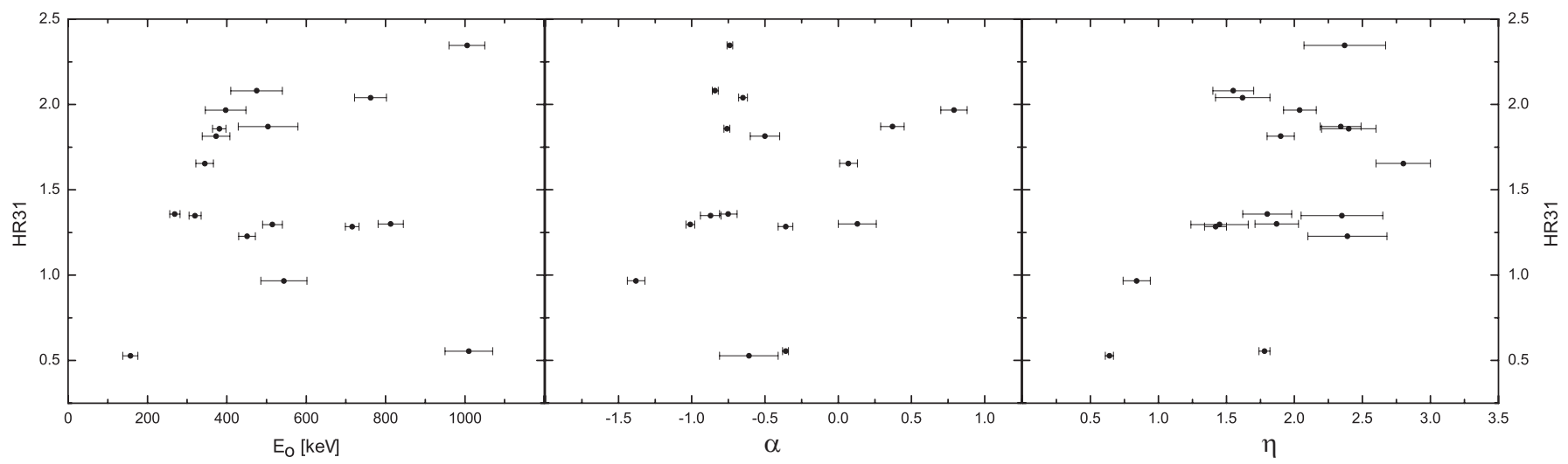

Fig. 5. Hardness ratio (HR31) as a function of $E_{0}, \alpha$, and $\eta$. Only weak correlations emerge, as expected.

\section{Discussion}

Interestingly, Eq. (2) shows that the observed photon (energy) flux of a pulse is inversely proportional to the spectral lag. Norris et al. (2000) similarly found that bursts with high peak-fluxes predominantly have small lags (their Fig. 2). However, the flux lag relation we find is a much tighter correlation than compared to the plots in Norris et al. (2000). Our analyses differ in several aspects. Norris et al. (2000) study a general sample (including bursts with lower peak fluxes) and 


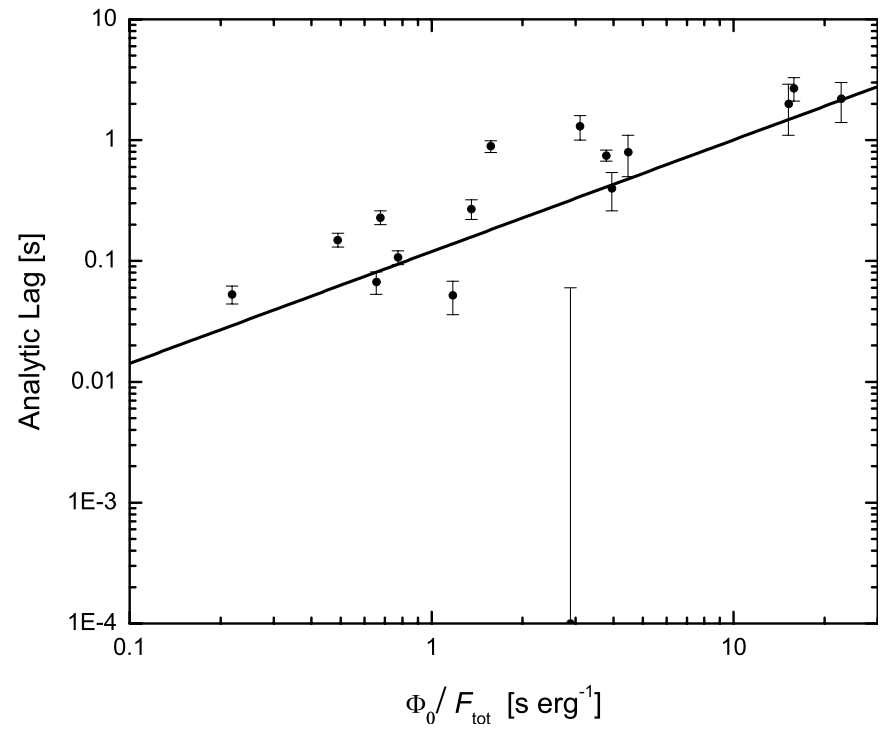

Fig. 6. Dependence of spectral lag on $\Phi_{0} / F_{\text {tot }}$. The outlier point is from the second pulse in trigger 973 .

not only single pulses. The lag value for multi-pulsed events will be an averaged value and the HR rather catches the distribution of $E_{0}$-values among the pulses in the studied burst. The general distribution in the flux-energy plane is furthermore determined by the HIC parameter $\eta$. Using bursts with a known redshift, Norris et al. (2000) translated their fluxes to luminosities and a stronger correlation emerged. More specifically, they found that the spectral lag, $\Delta t$, and the isotropically equivalent peak luminosity [or luminosity per steradian, that is, the power output of the burst assuming that the explosion occurs isotropically], $L / \Omega$, [erg/s] (see also Schaefer et al. 2001; Salmonson 2000) follows the relation

$L / \Omega=2.9 \times 10^{51} \mathrm{erg} / \mathrm{s}\left(\frac{\Delta t}{0.1 \mathrm{~s}}\right)^{-1.14 \pm 0.20}$.

Using the photon-number peak-luminosity [photons/s] instead, Salmonson (2000) found the exponent to be -0.98 , which all together is suggestive of inverse linear proportionality. We will in Sect. 3.1 below argue that Eq. (2) most probably is a consequence of this relation.

A positive correlation between the peak energy of the spectrum, $E_{\mathrm{p}}$, and the luminosity has been suggested empirically (Lloyd-Ronning \& Ramirez-Ruiz 2002; Amati et al. 2002). Combining this with the lag-luminosity anti-correlation (Eq. (3)) an anti-correlation is inferred between peak-energy and lag. This is indeed consistent with the behavior of the pulses in our sample, shown in Fig. 4d, especially for larger lags. The bursts with the longest lag have the lowest peak energies. Furthermore, the positive correlations between $\alpha$ and $\eta$ with lags (Figs. 4a and 4c) similarly imply that harder spectra (large $\alpha$ ) as well as steeper HICs (large $\eta$ ) are associated with bursts of lower luminosity.

We also noted that different pulses within a burst have different lags. Such a conclusion was similarly drawn by Hakkila et al. (2004). Finally, we also note that Norris et al. (2000) found that bursts with large HRs predominantly have small

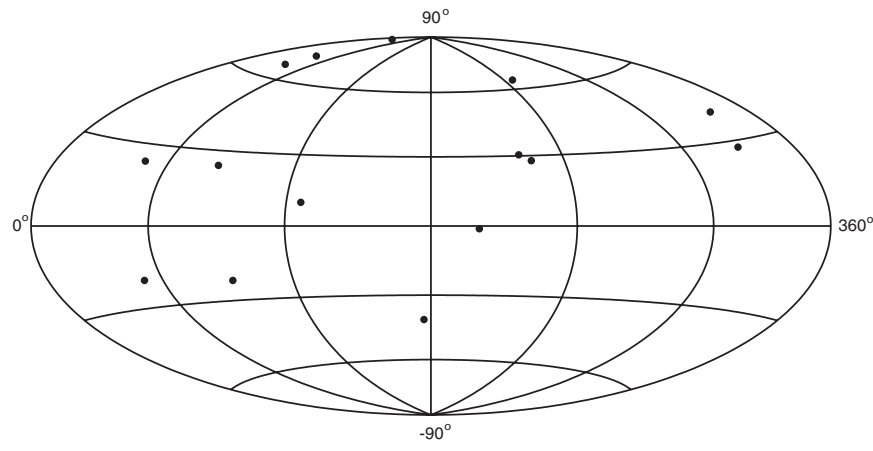

Fig. 7. Sky distribution in super-galactic coordinates for the 15 BATSE bursts in the studied sample. These are characterized by having long uncontaminated FRED pulse(s). The dipole moment of the distribution is very large.

lags. For our pulses in Table 2 we similarly find that the largest HR bursts mainly have small lags.

\subsection{Origin of the flux-lag correlation}

We find, in our sample, that the observed flux dispersion is $\sim 1.5$ orders of magnitude and follows a lag-flux power-law relation (Eq. (2)). Comparing it with the similar relation between the luminosity and lag of Norris et al. (2000) we can, in principle, have two different possibilities. First, the lag-flux relation is a consequence of the lag-luminosity correlation. In this case, the latter relation has to be valid for our sample as well. Second, Eq. (2) could be dominantly a consequence of the distribution of the 15 bursts in redshift. In this case, Eq. (4) may, but need not, be correct. Since the dispersion in the measured fluxes is 1.5 orders of magnitude, it is obvious that for the former case a smaller dispersion in $z$ is needed. Below, we will argue that it is indeed the lag-luminosity relation that is revealed.

The quantity measured by BATSE is the photon count rates and the derivation of the energy fluxes will by necessity be model dependent. Therefore it is more useful to use the more fundamental quantities given by the photon rates in discussing these matters. The photon luminosity, $L_{\text {iso }}$ [photon/s], that corresponds to a certain photon flux value, $F$ [photon $\left./ \mathrm{s} / \mathrm{cm}^{2}\right]$, observed from a source at a redshift, $z$, is given by (Carroll et al. 1992) (see also Mészáros \& Mészáros 1996)

$L_{\text {iso }}=\frac{4 \pi F}{1+z} d_{\mathrm{L}}^{2}$

$d_{\mathrm{L}}=\frac{(1+z) c}{H_{0}} \int_{0}^{z} \frac{\mathrm{d} z^{\prime}}{\sqrt{\left(1+z^{\prime}\right)^{2}\left(1+\Omega_{\mathrm{M}} z^{\prime}\right)-z^{\prime}\left(2+z^{\prime}\right) \Omega_{\Lambda}}}$

where $H_{0}$ is the Hubble constant, $\Omega_{\mathrm{M}}$ is associated with the present day matter density, $\Omega_{\Lambda}$ with the dark energy density, and $\Omega_{M}+\Omega_{\Lambda}=1$, that is, we consider a flat universe at once. The luminosity distance is denoted by $d_{\mathrm{L}}$. The extra $(1+z)$ factor in the denominator in Eq. (4) account for the fact that we are using the photon flux and not the energy flux which is assumed in the definition of $d_{\mathrm{L}}$ (see, e.g., end of Sect. 2 in Mészáros \& Mészáros 1995). Furthermore, in Eq. (4) we have neglected the K-correction, which takes into account the effect of $z$ on the 
bandpass. As shown by, for instance, Bloom et al. (2001), the quantitative distribution of fluxes does not change dramatically.

For the flux-lag correlation to emerge, the dispersion of the quantity $(1+z) / d_{\mathrm{L}}^{2}$ should then be smaller than $\sim$ two orders of magnitude. If this is the case, then the dispersion in luminosities should dominate the dispersion of flux. It has actually already observationally been confirmed that such dispersions exist. For the luminosities of bursts with known redshifts there is a dispersion of roughly two-to-three orders of magnitude (e.g., Fig. 6 of Mészáros 2001). Taking typical values of $\Omega_{M}=0.27$ and $\Omega_{\Lambda}=0.73$ (Riess et al. 2004)), and if the pulses originate in bursts that are, say, from $0.5<z<1.0$, then the ratio of minimal and maximal $d_{\mathrm{L}}$ is 5.5 , and hence $(1+z) / d_{\mathrm{L}}^{2}$ varies by a factor $\simeq 20$. Note that for this ratio the exact value of $H_{0}$ is unimportant. With such a distribution in $z$, the lag-luminosity relation of Eq. (4) will be dominant, and the lag-flux correlation will be observed. The actual distribution in $z$ is not known, but more arguments can be found to support such a range.

First of all, it must be recognized that, remarkably, the quantity $(1+z) / d_{\mathrm{L}}^{2}$ has the largest dispersion for the smallest $z$-values (Mészáros \& Mészáros 1988). If our analyzed bursts were at say $z \simeq 0.005$ one would thus expect a large dispersion diluting the lag-luminosity correlation. Furthermore, in the case of these small redshifts one would also expect a concentration of objects towards the galaxies in the local universe. These are distributed in a major planar structure centered around the Virgo cluster of galaxies, called the super-galactic plane, SGP (Peebles 1993; Lahav et al. 2000; Norris 2002). If the bursts in our sample were to be associated with these galaxies, their distribution in super-galactic coordinates would have a high quadropole moment, and the conclusion would be that they lie within a redshift of $z \sim 0.01$. We note here that only one GRB has been detected at a similarly small distance (GRB 980425/SN98bw, $z=0.0085$ ). We therefore plot in Fig. 7 the sky distribution of our sample in super-galactic coordinates. We calculated the dipole, $D=$ $\langle\cos (s g b)\rangle$, and quadropole, $Q=\left\langle\sin ^{2}(s g b)-1 / 3\right\rangle$ moments of the angular distributions. For the 15 bursts, $D=0.362$ (notably large) and $Q=0.0455$. These bursts are avoiding the south ssuper-galactic region, hence the large dipole moment. Since the belt around the super-galactical equator, up to $s g b= \pm 30 \mathrm{deg}$ in super-galactical latitudes, covers half the sky, one would expect a statistically significant over-density in this belt for objects associated with the SGP. However, the quadropole moment we find is relatively low; in the $s g b=$ \pm 30 deg-belt there are $8 \mathrm{GRBs}$, in the remaining part of sky 7 GRBs. In the BATSE exposure function, in the super-galactic plane, there will be higher, non-vanishing moments, albeit they will be small, and at least for 15 events the shot noise will cover them. We also produced 1000 Monte-Carlo catalogues with 15 events to test the robustness of this result. These catalogues follow the BATSE sky exposure. If we order the simulated distributions from 1 to 1000 in increasingly anisotropic distributions, the observed dipole value lies at 993 , that is, the confidence limit is about $99.3 \%$. Our sample is thus not strongly distributed along the super-galactic plane and we therefore conclude that our sample is not completely within this distance, but beyond it. In this connection it should be noted that
Norris (2002) studied a (totally different) sample of longlagged burst and plotted them in super-galactic coordinates and did indeed find a large quadropole moment. This led him to suggest that his sample is associated with $\mathrm{SNe}$ type $\mathrm{Ib} / \mathrm{c}$ in the local super cluster of galaxies.

Second, very large $z$-values for the $15 \mathrm{GRBs}$ in our sample can also be excluded. Our sample is limited to the brightest BATSE bursts ( $>2$ photons $/ \mathrm{s} / \mathrm{cm}^{2}$ ) and to cases with a single or a few well shaped pulses. The first constrain leaves bursts from the BATSE catalogue which are normally totally dominated by lags which are very short ( $\$ 250 \mathrm{~ms}$; see Fig. 2 in Norris 2002). According to Eq. (3) these short-lag bursts correspond to luminous explosions leading to the high fluxes observed. The Norris (2002) sample also has the property that bursts with lags greater than $\sim 3 \mathrm{~s}$ all have peak fluxes lower that 2 photons $/ \mathrm{s} / \mathrm{cm}^{2}$. However, the measured lags in our sample (Table 4) are dominated by long lags and are thus low-luminosity bursts (according to Eq. (3)). Following the nomenclature introduced by Norris (2002) these thus constitute a fourth region in the peakflux-lag plane, namely the bright and long-lag bursts. Since they are low-luminous bursts but still bright this means that they cannot be very distant. The second constraint that we used excludes the most variable bursts. According to the empirical correlation between variability and luminosity (Reichart et al. 2001) this again leaves low-luminosity bursts. To be able to study individual pulses we need bursts of low variability which are also bright. These criteria left us with this sample.

Third, by assuming the lag-luminosity relation to be valid for our sample, we can calculate a luminosity for each pulse and, in combination with the measured flux value, we can arrive at an estimation of the redshift. These estimated redshifts are given in the last column in Table 3 . The redshifts are all below $z \sim 1.8$ except trigger 7293, which is derived to have $z=8.6$. The peak of the distribution is at $z \sim 0.7$. In any case, this also supports the small dispersion of $(1+z) / d_{\mathrm{L}}^{2}$. Note here the obvious discrepancy in redshifts for the multi-peaked events which arises due to the variation in lags, already pointed out in Paper I and above. The sample studied is therefore most probably from a relatively limited distance range (low dispersion in $z$ ) at not very high redshifts, but mainly beyond $z \sim 0.01$.

Another argument that supports the contention that the correlations in Eqs. (2) and (4) are the same comes from the cosmological time-dilation. Let us rewrite Eq. (4) in the form

$L / \Omega=L^{\prime}\left(t_{0} / \Delta t_{\mathrm{rf}}\right)^{\alpha}$

where $L^{\prime}=2.9 \times 10^{51} \mathrm{erg} / \mathrm{s}, t_{0}=0.1 \mathrm{~s}$ and $\alpha \simeq 1$. For our purpose it is essential that $\alpha>0$, and the fact that $\Delta t_{\mathrm{rf}}$ is the rest-frame lag. Then the observed flux will be given by $F=$ $L^{\prime}\left(t_{0} / \Delta t_{\mathrm{rff}}\right)^{\alpha}(1+z) / d_{\mathrm{L}}^{2}=L^{\prime}\left(t_{0} / \Delta t_{\mathrm{obs}}\right)^{\alpha}(1+z)^{1+\alpha} / d_{\mathrm{L}}^{2}$, since $\Delta t_{\mathrm{obs}}=$ $(1+z) \Delta t_{\mathrm{rf}}$. This means that one should consider the dispersion of $(1+z)^{1+\alpha} / d_{\mathrm{L}}^{2}$ instead of $(1+z) / d_{\mathrm{L}}^{2}$. A positive $\alpha$ value will decrease the effect of the distance dispersion on the correlation.

In summary, all this suggests that the correlation between the observed fluxes and lags (Eq. (2)) is a residual of the lag-luminosity correlation (Eq. (3)) that is still apparent. 


\subsection{Origin of the spectral correlations}

Several observational facts appear to indicate that long GRBs originate when the iron-core of a massive star collapses to a black hole or a rapidly rotating neutron star, producing a collimated, relativistic jet (collapsar model; see Woosley 1993). The collimation angle of the outflow varies, which leads to different behaviors of the observed light curves. The observer is assumed to see the GRB approximately along the jet axis and the opening angle of the collimation of the outflow, $\theta_{\mathrm{j}}$, may vary and is responsible for the diversity in the quantity $L / \Omega$. Since $L / \Omega$ is observed to be correlated to the variability of the $\gamma$-ray light curve, $V$ (Reichart et al. 2001), a $V-\theta_{\mathrm{j}}$-correlation should also exist. This was indeed shown to exist by Kobayashi et al. (2002), who used bursts for which an opening angle had been deduced. They also suggested an explanation of the correlations within the internal shock model. There are two possible smoothing effects and they both dependent on the bulk Lorentz factor: the location of the pair photosphere produced by synchrotron photons at $R_{ \pm} \sim 1 / \sqrt{\Gamma}$ and the angular time-scale $t_{\text {ang }} \sim R / 2 c \Gamma^{2}$. If a collision happens below the photosphere, the whole internal energy produced by the collision is converted to kinetic energy again via the shell spreading. It is only collisions that occur above the photosphere that can be seen by an observer. For instance, a small $\Gamma$ yields a large photospheric radius and the outflow then becomes more ordered and therefore the variability is lower. In the same manner, a small $\Gamma$ yields a large $t_{\text {ang }}$ and thus a less variable light curve. Therefore, the authors argued that the variability is determined mainly by the bulk $\Gamma$ of the outflow and thus the $V-\theta_{\mathrm{j}}$ correlation reduces to a $\Gamma-\theta_{\mathrm{j}}$ correlation. Simulating the hydrodynamic collisions numerically, Kobayashi et al. (2002) found that the data are fitted the best with $\Gamma \propto \theta_{j}^{-1}$ and thus the mass loading $M \propto E /\left(c^{2} \Gamma\right) \propto \theta$, since the total explosion energy has been found to be approximately the same from burst to burst (e.g., Frail et al. 2001). This scenario is reasonable in the collapsar model: a wide jet leads to a dim burst since a wider jet involves more mass at the explosion, leading to lower speeds of the outflowing material. The change in $L / \Omega$ therefore depends mainly on $\theta_{\mathrm{j}}$. The variability, $V$, is determined by the opening angle which in its turn also affects the quantity, $L / \Omega \propto L_{\text {const }} / \theta_{\mathrm{j}}^{2}$. In this model $L \propto \Gamma^{2}$.

If the spectral lag is also determined by the angular spreading time scale, which is the key time scale in this model, the lag-luminosity relation can be found. In this model luminosity is scaled by the opening angle $L \propto \theta^{-2}$, while the angular spreading time is $t_{\text {ang }} \propto \Gamma^{-2} \propto \theta^{2}$. This leads to $L \propto t_{\text {ang }}^{-1}$, which is Eq. (3). According to Paper I, the lag is mainly determined by the pulse time scale $T$, which is defined as $T=\Phi_{0} E_{0} / F_{0}$. Since lag and luminosity are correlated, $\Phi_{0} /$ flux should also be approximately correlated, as shown in Fig. 6.

In the description above it is assumed that the variations in Lorentz factors are mainly due to variations in the collimation opening-angle of the outflow, and that the jets have a uniform profile out to the opening angle. However, an alternative scenario, for which the discussion above is also applicable, is the structured jet in which a certain beam profile is assumed (e.g. Rossi et al. 2002). In such a case the most luminous part of the jet has a higher Lorentz factor. A large jet opening angle gives the possibility for large off-axis views. The probability for a certain viewing angle is indeed proportional to the viewing angle itself, and thus large angles are favored. If there is a typical jet profile in all bursts, larger opening angle outflows are most often seen more off-axis, with a lower $\Gamma$ as a consequence.

Ryde (2004) found, by analyzing spectrally hard BATSE pulses, that the spectra can be modelled by a thermal black-body superimposed on a non-thermal, optically-thin synchrotron spectrum (see also Ghirlanda et al. 2003). The relative strengths of these two components vary. A large $\alpha$-value is measured if the thermal component is dominant, while a lower value, more like a synchrotron value, is found if it is less prominent and the non-thermal component dominates. Small outflow velocities, $\Gamma$, lead to larger photospheric radii, and less dissipation of energy in shocks (for example) which enhances the thermal emission, thus leading to a harder $\alpha$, as well as larger lags. So, a low $\Gamma$ leads to low luminosity bursts (due to larger photospheric radii, as well as lower $\Gamma$ ) and hard spectra. This is indeed the trend in Fig. 4a, assuming that the lag can be translated to luminosity through the lag-luminosity relation. Here, that relation is observationally based in Fig. 2 and all the relations in Fig. 4 then correspond to relations with luminosity. A pure black-body emission should have a HIC of $\eta=4$. If the photospheric emission is dominant, a high $\eta$ should be correlated with a lower luminosity (Fig. 4c). This also provides a natural explanation for a $\alpha-\eta$ correlation in Fig. 4b.

Further, Fig. 4d suggests that for low-luminosity bursts there is a positive luminosity-to-peak-energy correlation. For the smallest lags (highest luminosities) the peak-energies decrease and there is an anti-correlation. In the optically-thin synchrotron-shock model an anti-correlation arises most naturally. $E_{\mathrm{p}} \propto \Gamma B^{\prime} \gamma_{\mathrm{e}}^{2}$, where $B^{\prime}$ is the comoving magnetic-field strength and $\gamma_{\mathrm{e}}$ is the characteristic Lorentz factor of an emitting electron, and since, in the internal shock model, $\gamma_{\mathrm{e}}^{2}$ is mainly dependent on the relative Lorentz factor of the colliding shells and not on $\Gamma, E_{\mathrm{p}}$ is proportional to $\Gamma B^{\prime}$. This is the Lorentz-boosted magnetic field strength, of which the square should be proportional to the fireball energy density in the observer frame, $U \propto L / R^{2}$, where $R \propto \Gamma^{2}$ is the typical collision radius. Here we have assumed that the magnetic energy density is a constant fraction of the total energy density. Therefore we have $E_{\mathrm{p}} \propto L^{1 / 2} R^{-1} \propto L^{1 / 2} \Gamma^{-2}$. With, for instance, $L \propto \Gamma^{2}$ from above, we have $E_{\mathrm{p}} \propto L^{-1 / 2}$, that is, an anti-correlation. This is for synchrotron radiation. The peak energy of inverse-Compton scattered photons is at $E_{\mathrm{p}}^{\mathrm{IC}}=\gamma_{\mathrm{e}}^{2} E_{\mathrm{p}}^{\mathrm{synch}}$ and thus it has the same dependence. Zhang \& Mészáros (2002) also discussed the luminosity-peak-energy correlation for various other outflow models: For Poynting-flux dominated models $E_{\mathrm{p}} \propto \Gamma$, while for emission from the baryon photosphere, during the shell acceleration phase, $E_{\mathrm{p}} \propto L^{1 / 4}$. The last two outflows thus easily reproduce positive correlations. Furthermore, Ryde (2004) motivated that the time-evolution of the temperature decay of the thermal pulses they studied can be explained either by a baryonic photosphere during the acceleration phase or by a Poynting-flux dominated wind. In both these models a positive correlation is, as mentioned, expected. We can therefore interpret Fig. 4, following the suggestions in Ryde (2004). 
The positive correlation for low luminosities is for pulses that are dominated by the thermal emission. The negative correlation (at small lags) are then from pulses in which the nonthermal, synchrotron component dominates and thermal emission is less dominant. This is indeed consistent with Fig. 4a in which the small lag pulses have soft spectra, while the large lag cases have harder spectra.

This simple description can thus accommodate the main correlations and trends presented above. A burst does not necessarily need to have the same lag for all pulses within it, since it is mainly internal properties that determine the characteristics.

\subsection{Alternative models}

Throughout this paper it has been emphasized that the $E_{\mathrm{p}}$ evolution has an important role in determining the spectral lags. The HFC, $\dot{E}_{\mathrm{p}}=-F / \Phi_{0}$, which governs this evolution, could be due to thermal processes like saturated Comptonization without extra heating, which is difficult to achieve in general however. Such a scenario has been discussed by Liang \& Kargatis (1996) and Liang (1997). Schaefer (2004) argued further that the lag-luminosity relation can be explained by this behavior. This model assumes an impulsive heating of a confined plasma which starts to cool. There should not be any continuous heating, for example, dissipation of energy through shocks. Furthermore, there has to be a constant injection rate of soft photons which gives rise to the cooling of the electrons, $\dot{T}_{\mathrm{e}}$. The thermal emission ensures that the emitted photons have approximately the same energy as the averaged electron. The change of internal energy of the cloud $\dot{U}$, which gives the luminosity, is then $L=\dot{U}=N \dot{T}=N \dot{E}_{\mathrm{p}}$, where $N$ is the number of electrons. This equation is consistent with the HFC. Assuming, first, that $\mathrm{d} E_{\mathrm{p}} / \mathrm{d} t$ is constant for a pulse (which is not the exact case, see Ryde \& Svensson 2002), second, that the peak energy decays within typical range, that does not differ greatly from burst to burst (clearly also an approximation), and, third, that the peak luminosity, $L_{\text {peak }}$ is a good measure of the pulse luminosity, $L$, then the analytical finding in Paper I that

$\Delta t=\int_{\Delta E} \frac{\mathrm{d} t}{\mathrm{~d} E_{\mathrm{p}}} \mathrm{d} E$

combined with the HFC, gives that the lag

$\Delta t \propto-\frac{\Phi_{0}}{L_{\text {peak }}}$.

$\Phi_{0}$ is practically constant between pulses, and in the model above it is a combination of $N$, distance, and jet opening angle. This is similar to the lag- $\Phi_{0}$ correlation, discussed by Kocevski \& Liang (2003). This model has several advantages but needs a number of approximations and strong assumptions to hold.

As mentioned above the lag-luminosity relation is intriguingly close to a pure proportionality between the luminosity and the inverse of the lag. Salmonson (2000) noted that such a relation follows from simple relativistic kinematics if one assumes a common comoving time, which could be due to cooling or deceleration. Assuming first that the viewing angle (between the jet velocity and the line-of sight, l.o.s.) $\theta$ is not large, the angle-dependent Lorentz factor $\mathcal{D}(\theta)$ can be approximated by $2 \Gamma$ and the photon-number luminosity goes as $F_{N}=\Gamma F_{N}^{\prime}(1+z)^{-1}$, where $F_{N}^{\prime}$ is the comoving value. Since a typical comoving time scale, $\delta t$ goes as $\delta t^{\prime}(1+z) / 2 \Gamma$, the observed lag-luminosity relation emerges. The dominant effect in the dispersion of lags is assumed to be due to the value of $\Gamma$ directed towards the observer. This could be explained in two different ways. First, it is natural to assume that the Lorentz factor is different from burst to burst. Second, if the outflow is in the form of a jet, the flow velocity could very well be dependent on the angle from its axis, as mentioned above. If the l.o.s. is along the jet axis a large $\Gamma$ is observed, while if the l.o.s. is different from the jet axis the flow velocity might be significantly lower. The transformation of the time-scale and the flux will depend on the viewing angle, since they are affected by the angle-dependent Lorentz factor, $\mathcal{D}(\theta)$. Ioka \& Nakamura (2001) even argued that it is indeed the viewing angle that causes the dispersion in spectral lags and they showed that they could reproduce the observed relation between the peak luminosity and lags.

Daigne \& Mochkovitch (2003) presented a pulse description within the internal shock model, which can reproduce the lag-luminosity relation. The peak energy is parameterized as $E_{\mathrm{p}} \propto \rho^{x} \epsilon^{y} \Gamma$, where $\rho$ is the post-shock density and $\epsilon c^{2}$ is the post-shock dissipated energy per unit mass. Standard synchrotron radiation, assuming classical equipartition, has $x=$ $1 / 2$ and $y=5 / 2$. However, if the equipartition between the magnetic field and particle energies vary with either $\rho, \epsilon$, or both, then other values of $x$ and $y$ can exist. The authors found that the observed spectral pulse behavior is best reproduced with $x=y=1 / 4$. Their model also predicts that the hardnessratio should decrease with spectral lag, which is similar to what is found by Norris et al. (2000).

\section{Conclusions}

We have studied the spectral lags in a sample of bright pulses, all belonging to the class of long duration bursts (see, e.g. Balázs et al. 2003; Horváth 1998). We find that there is a large spread in the measured lag values. In particular we find that the lag values differ considerably between pulses within a burst. This is important to note, since often the whole light curve is used to measure the spectral lag, and the lag measurement then by necessity is only an averaged value. Also, models which cannot accommodate a variable lag over a pulse, that is, models which rely solely on external effects, need to be refined.

We also find that a correlation between the observed flux and the spectral lag is valid for prominent pulses, very similar to the lag-luminosity relation that has previously been reported for entire bursts, that is, not only for pulses. We draw the conclusion that the observed relation is a residual of the physical lag-luminosity relation. The sample does not show a significantly strong clustering towards the super-galactic plane, which indicates that the bursts are at a larger distance than $z \sim 0.01$. However, they cannot lie at very large distances, since they are mainly low-luminous and bright bursts.

We have compared the analytic lags with the values found by using the CCF. The CCF underestimates the lags somewhat, 
but there is a linear relation between the measurements. Also we find that the results found by using the count light-curves and the photon-flux light-curves do not differ significantly and thus permit their use.

The observed correlations are similar to the general analytical and numerical simulation results in Paper I. Foremost we find that the lag correlates strongly with the decay time-scale of the pulse and that the relation is linear. This means that the relationships including the lag, that have been identified, should be translatable to relationships including the pulse time-scale. This time-scale is closely connected to the creation process and radiation processes in the out-flowing plasma.

We also argue for a collapsar model scenario, where the collimation is the important factor leading to variation in the observed parameters. The observations are consistent with the existence of several components in the spectra, thermal and non-thermal emission, varying in relative strength.

Acknowledgements. We are grateful to Drs. M. Briggs and R. Preece for valuable support. We also thank Drs. C.-I. Björnsson, S. Kobayashi, and P. Mészáros for interesting discussions. The anonymous referee is acknowledged for useful comments that improved the manuscript. This study was supported by the Swedish Research Council and the Hungarian OTKA grant No. T034549, by the research plan J13/98: 113200004 of the Czech Ministry of Education, Youth and Sports, and by a grant from the Wenner-Gren Foundations (A.M.). This research made use of data obtained through the HEASARC Online Service provided by NASA's Goddard Space Flight Center. F.R. wishes to express his gratitude to the host Departments in Prague and Budapest for their kind hospitality during his visits.

\section{References}

Amati, L., Frontera, F., Tavani, M., et al. 2002, A\&A, 390, 81

Band, D., Matteson, J., Ford, L., et al. 1993, ApJ, 413, 281

Balázs, L. G., Bagoly, Z., Horváth, I., Mészáros, A., \& Mészáros, P. 2003, A\&A, 401, 129

Band, D. L. 1997, ApJ, 486, 928

Bloom, J. S., Frail, D. A., \& Sari, R. 2001, AJ, 121, 2879
Borgonovo, L., \& Ryde, F. 2001, ApJ, 548, 770

Carroll, S. M., Press, W. H., \& Turner, E. L. 1992, ARA\&A, 30, 499

Daigne, F., \& Mochkovitch, R. 2003, MNRAS, 342, 587

Fishman, G., et al. 1989, in Gamma Ray Observatory Science Workshop, ed. W. N. Johnson (Washington: NASA), 2

Frail, D. A., Kulkarni, S.R., Sari, R., et al. 2001, ApJ, 562, L55

Ghirlanda, G., Celotti, A., \& Ghisellini, G. 2003, A\&A, 406, 879

Hakkila, J., \& Giblin, T. W. 2004, ApJ, in press [arXiv:astro-ph/0403360]

Horváth, I. 1998, ApJ, 508, 757

Ioka, K., \& Nakamura, T. 2001, ApJ, 554, L163

Kobayashi, S., Ryde, F., \& MacFadyen, A. 2002, ApJ, 577, 302

Kocevski, D., \& Liang, E. 2003, ApJ, 594, 385

Kocevski, D., Ryde, F., \& Liang, E. 2003, ApJ, 596, 389

Lahav, O., Santiago, B. X., Webster, A. M., et al. 2000, MNRAS, 312, 166

Liang, E., \& Kargatis, V. 1996, Nature, 381, 49

Lloyd-Ronning, N. M., \& Ramirez-Ruiz, E. 2002, ApJ, 576, 101

Mészáros, P. 2001, Science, 291, 79

Mészáros, A., \& Mészáros, P. 1988, ApJ, 327, 25

Mészáros, A., \& Mészáros, P. 1996, ApJ, 466, 29

Mészáros, P., \& Mészáros, A. 1995, ApJ, 449, 9

Norris, J. P. 2002, ApJ, 579, 386

Norris, J. P., Marani, G. F., \& Bonnell, J. T. 2000, ApJ, 534, 248

Peebles, P. J. E. 1993, Principles of Physical Cosmology (Princeton: Princeton University Press)

Reichart, D. E., Lamb, D. Q., Fenimore, E. E., et al. 2001, ApJ, 552, 57

Riess, A. G., Strolger, L., Tonry, J., et al. 2004, ApJ, 607, 665

Rossi, E., Lazzati, D., \& Rees, M. J. 2002, MNRAS, 332, 945

Ryde, F. 2004, ApJ, 614, 827

Ryde, F. 2005, A\&A, 429, 869

Ryde, F., \& Svensson, R. 2000, ApJ, 529, L13

Ryde, F., \& Svensson, R. 2002, ApJ, 566, 210

Salmonson, J. D. 2000, ApJ, 544, L115

Schaefer, B. E. 2004, ApJ, 602, 306

Schaefer, B. E., Deng, M., \& Band, D. L. 2001, ApJ, 563, L123

Woosley, S. E. 1993, ApJ, 405, 273

Wu, B., \& Fenimore, E. 2000, ApJ, 535, L29

Zhang, B., \& Mészáros, P. 2002, ApJ, 581, 1236 